\title{
Evaluation of a high sensitivity thyroglobulin assay for use in patients following total thyroidectomy and radioiodine ablation
}

\author{
Background \\ -Thyroglobulin $(\mathrm{Tg})$ is used for monitoring patients who have undergone total thyroidectomy (TT) and radioiodine (RAI) ablation therapy for thyroid cancer. \\ -The current method is the Siemens Immulite assay with limit of quantification of $2 \mathrm{ng} / \mathrm{mL}$ following in-house evaluation. \\ - Recent guidelines suggest the use of high sensitivity $\mathrm{Tg}(\mathrm{hs}-\mathrm{Tg}$ ) as an alternative to TSH stimulated Tg levels. \\ - The aim is to evaluate the hs-Tg Beckman Access II assay with a stated functional sensitivity of $0.1 \mathrm{ng} / \mathrm{mL}$.
}

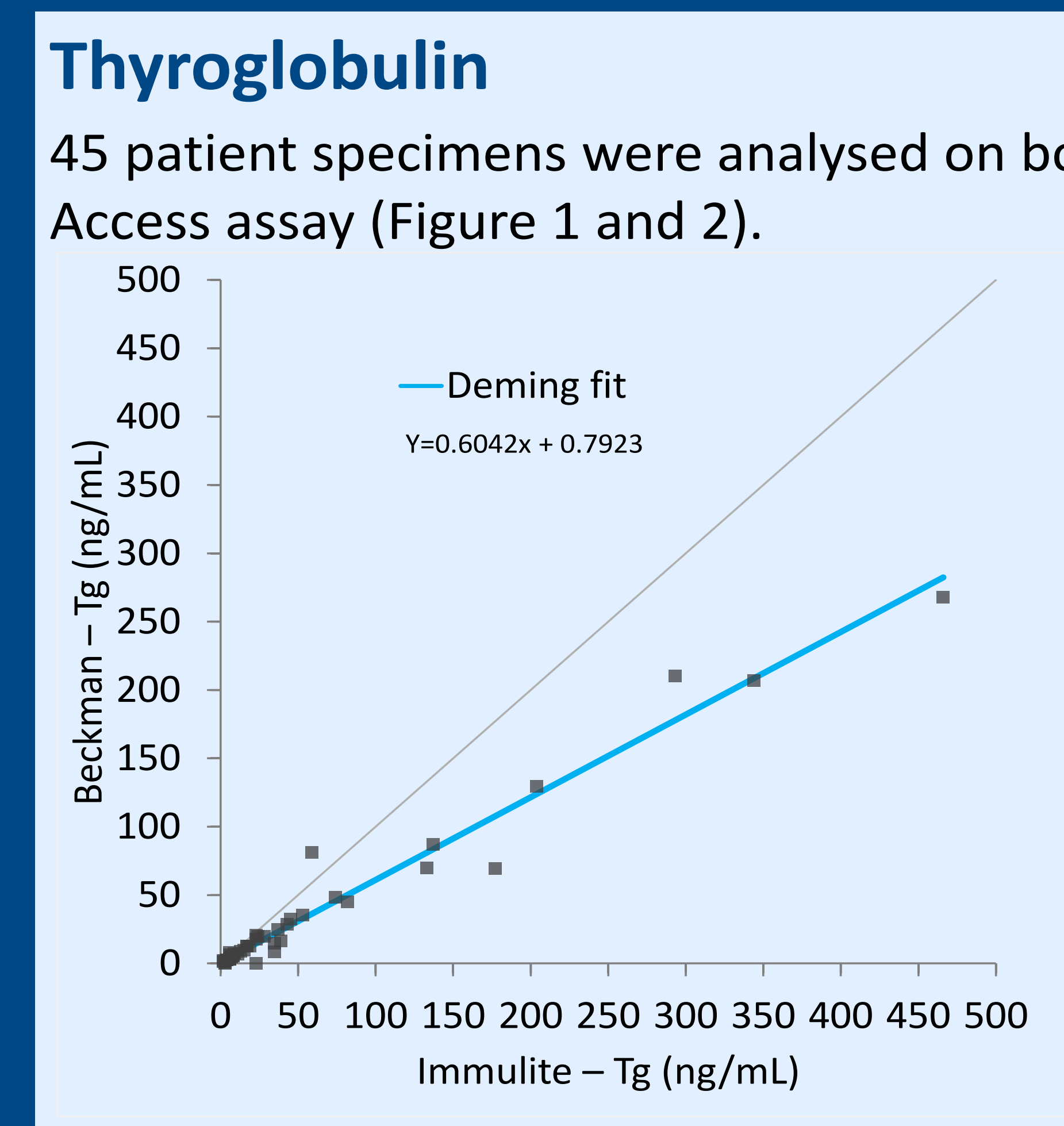

Figure 1. Deming regression of patient data

-The Beckman hs-Tg assay showed acceptable EQA performance compared to UK NEQAS method mean.

-Imprecision was acceptable for both patient samples (table 1) and IQC material.

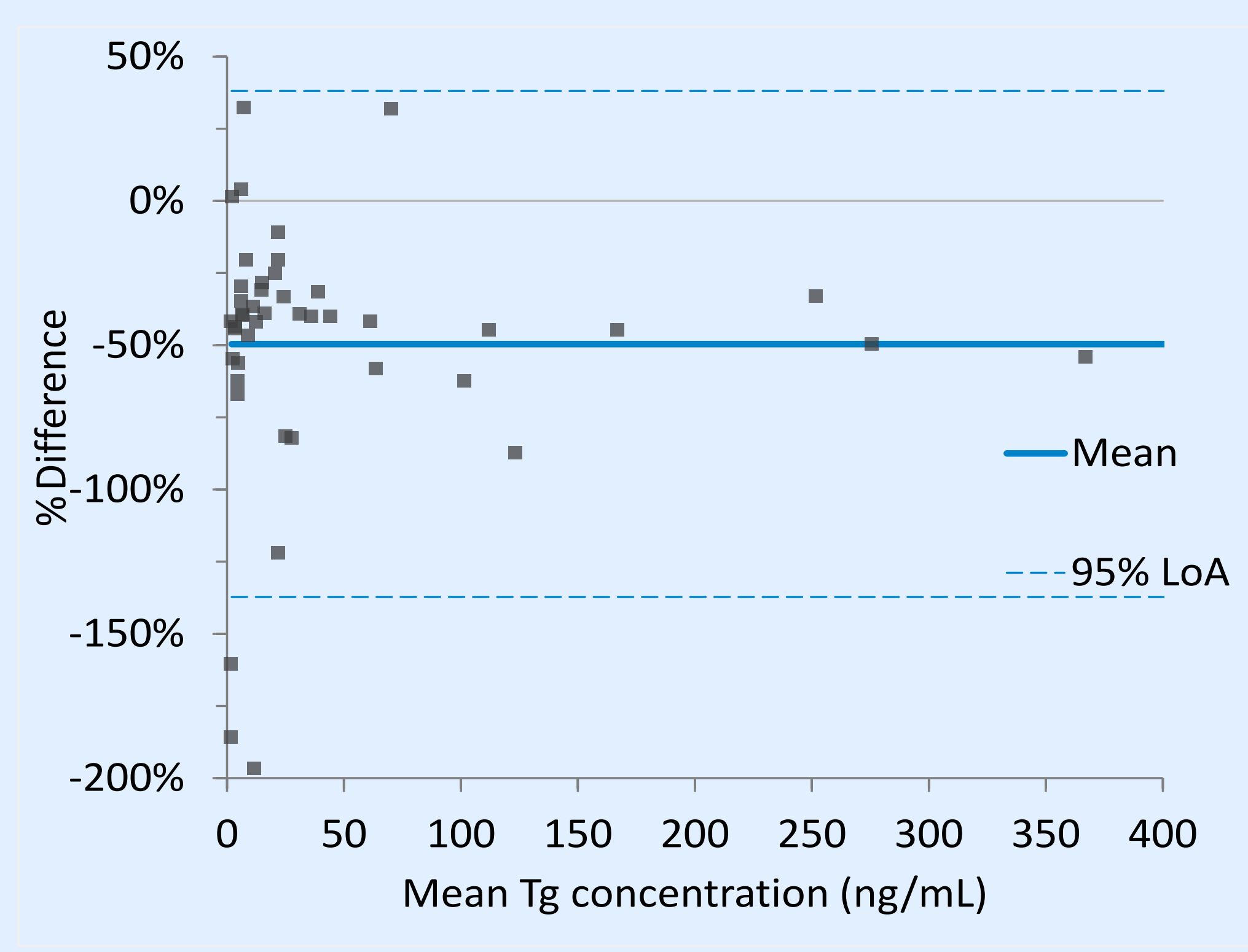

Figure 2. Bland-Altman plot of patient data

\begin{tabular}{|c|c|c|}
\hline $\begin{array}{l}\text { Mean } \\
(\mathrm{ng} / \mathrm{mL})\end{array}$ & $\begin{array}{l}\text { Intra-assay } \\
\text { imprecision } \\
\text { (\%CV) }\end{array}$ & $\begin{array}{l}\text { Total imprecision } \\
(\% \mathrm{CV})\end{array}$ \\
\hline 0.13 & 10.2 & 11.3 \\
\hline $0.77^{*}$ & 2.0 & 3.6 \\
\hline 1.13 & 2.4 & 11.3 \\
\hline 2.62 & 1.7 & 7.3 \\
\hline
\end{tabular}

\section{Thyroglobulin antibodies}

Guidelines recommend measurement of thyroglobulin antibodies on all thyroglobulin requests. The Beckman TgAb assay was evaluated against the Immulite assay (Figure 3).

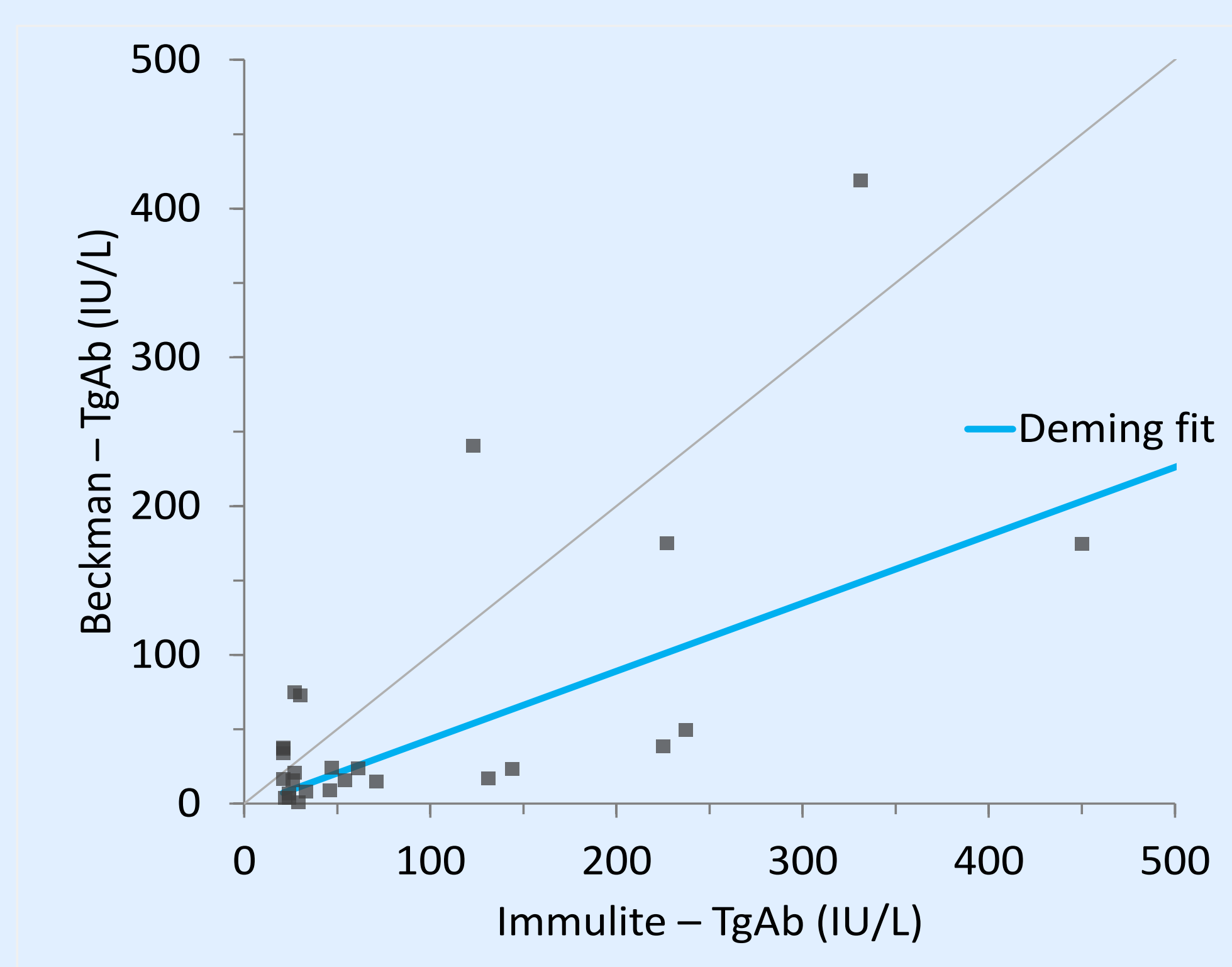

Figure 3. Deming regression of patient data

-The Beckman TgAb assay showed acceptable EQA performance compared to the method mean.

-Imprecision was within manufacturers stated performance (Tables 2 and 3).

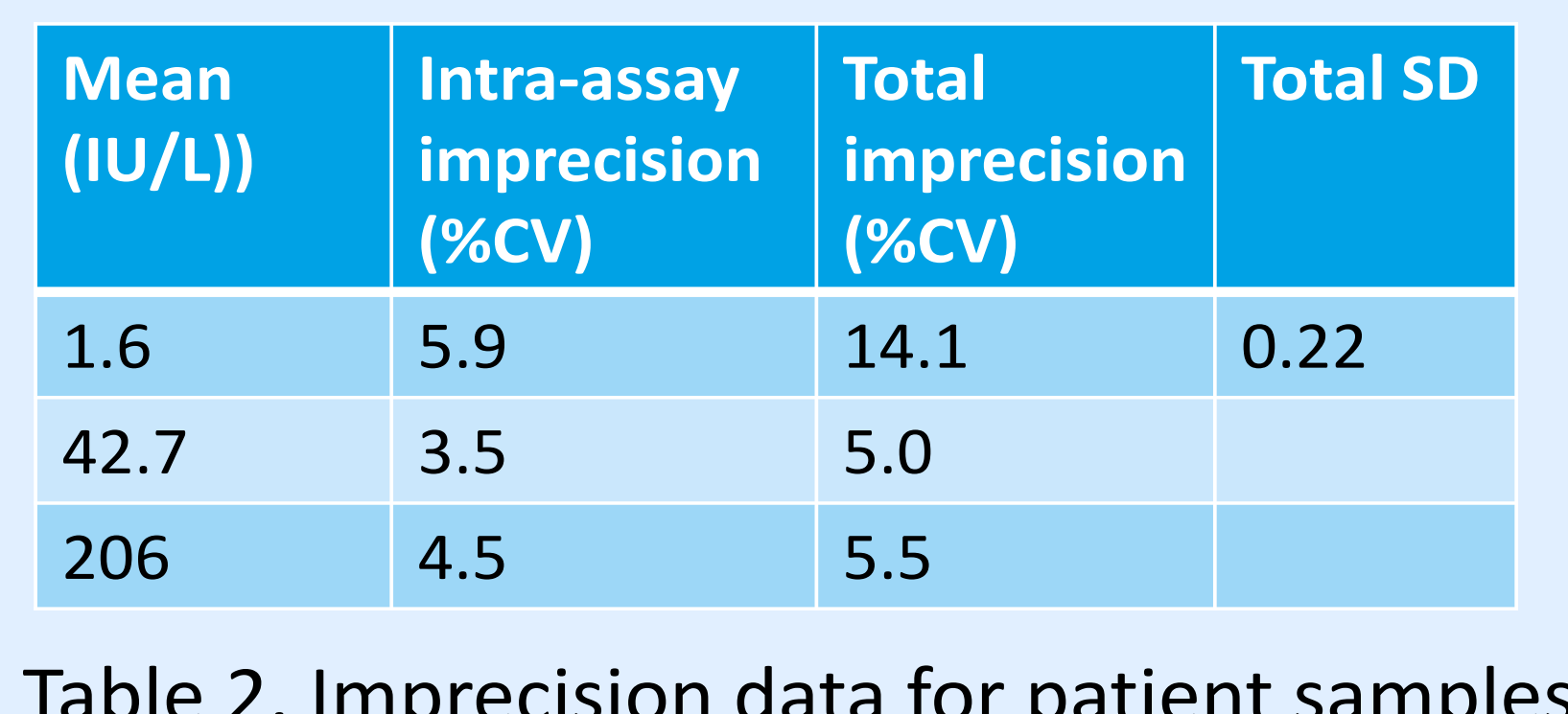

Table 2. Imprecision data for patient samples

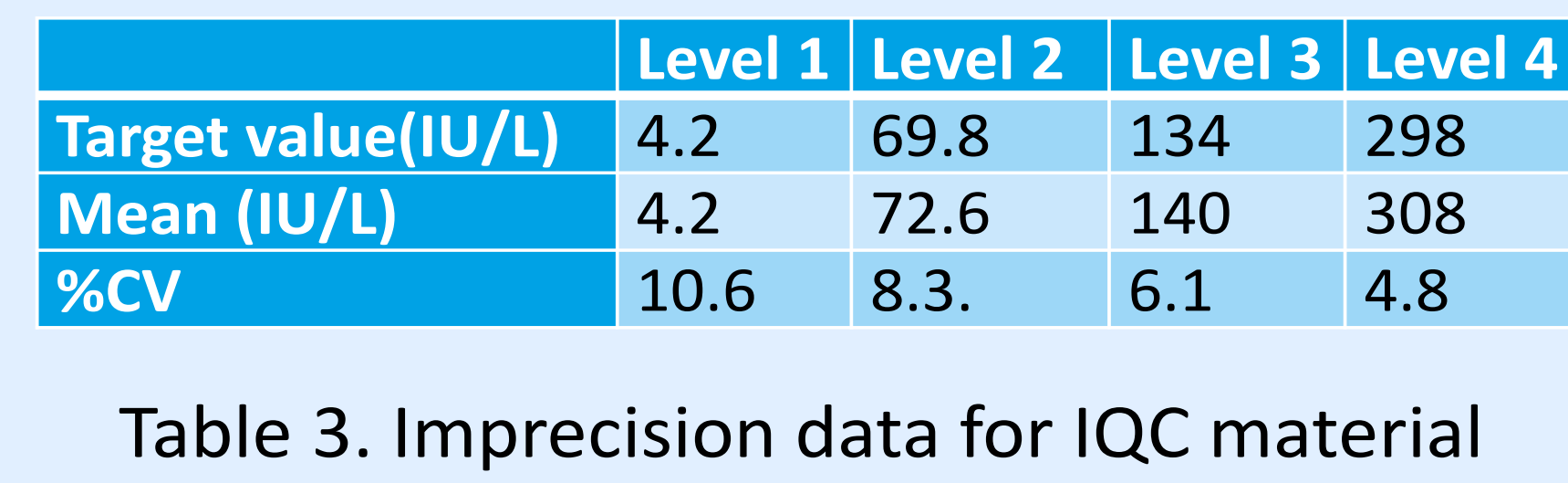

\section{Clinical evaluation}

Method

-Samples were analysed from 140 patients with an Immulite $\mathrm{Tg}<2 \mathrm{ng} / \mathrm{mL}$ and $\operatorname{TgAb}<20 \mathrm{IU} / \mathrm{L}$ following TT and RAI.

- Clinical information obtained from electronic patient records to include time of TT and RAI Hs-Tg results

- Results were classified as detectable or undetectable based on the quoted functional sensitivity of $0.1 \mathrm{ng} / \mathrm{mL}$.

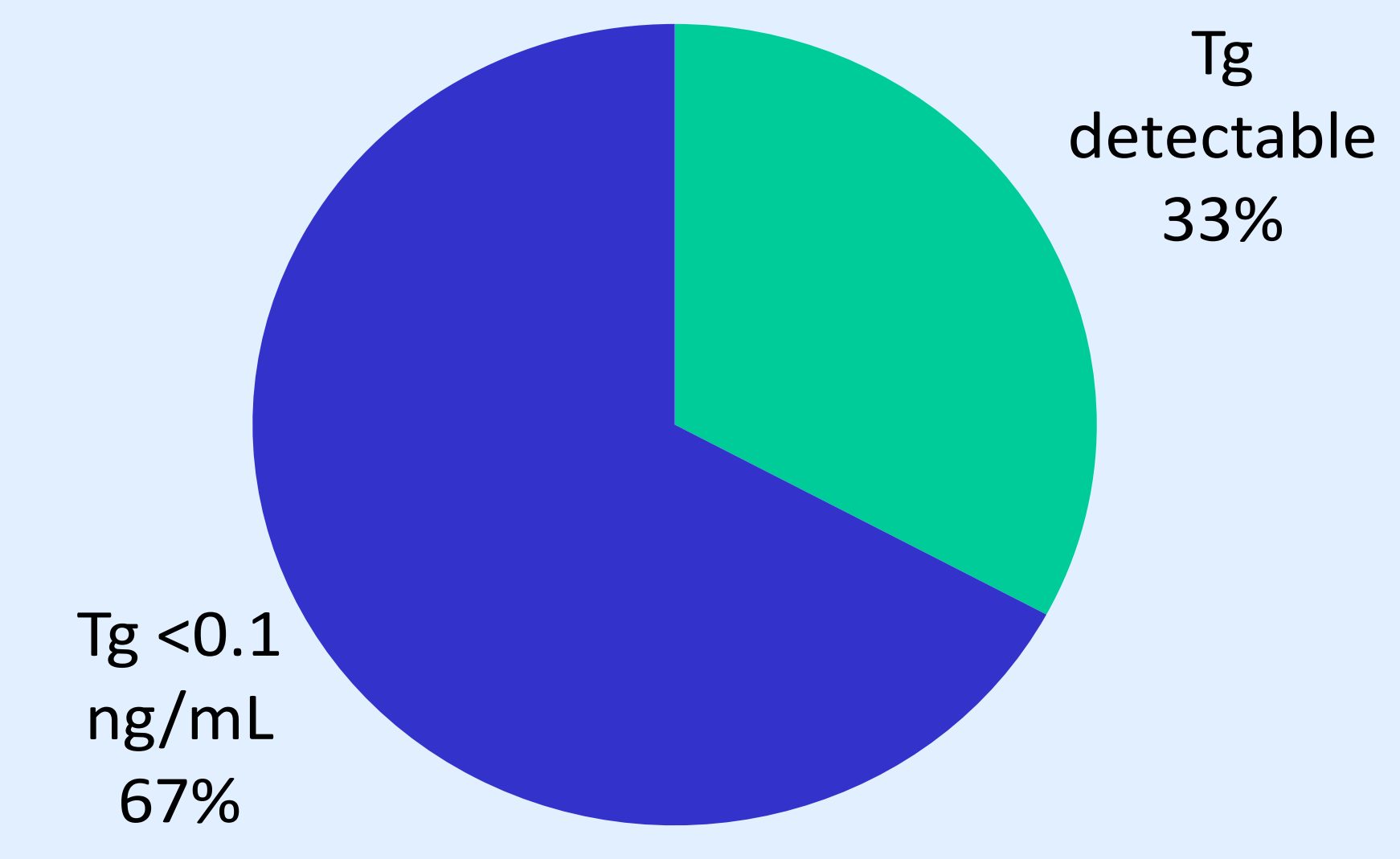

Figure 4. Frequency of thyroglobulin result by classification

Time post RAI treatment

- Time post RAI was obtained for all patients and ranged from one month to twenty years. -Median time post RAI was four years.

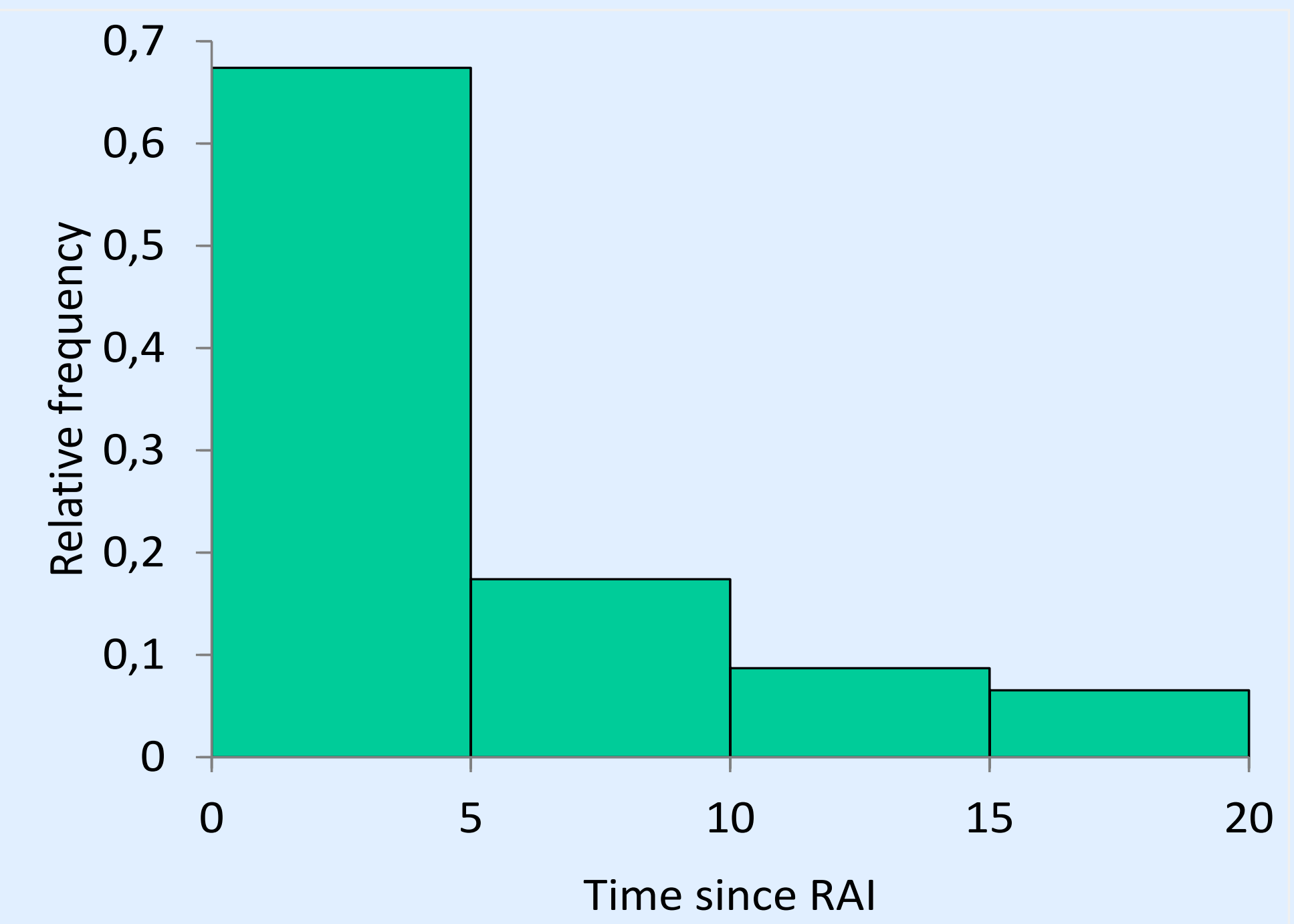

Figure 5. Time post RAI treatment in patients with a detectable hs-Tg

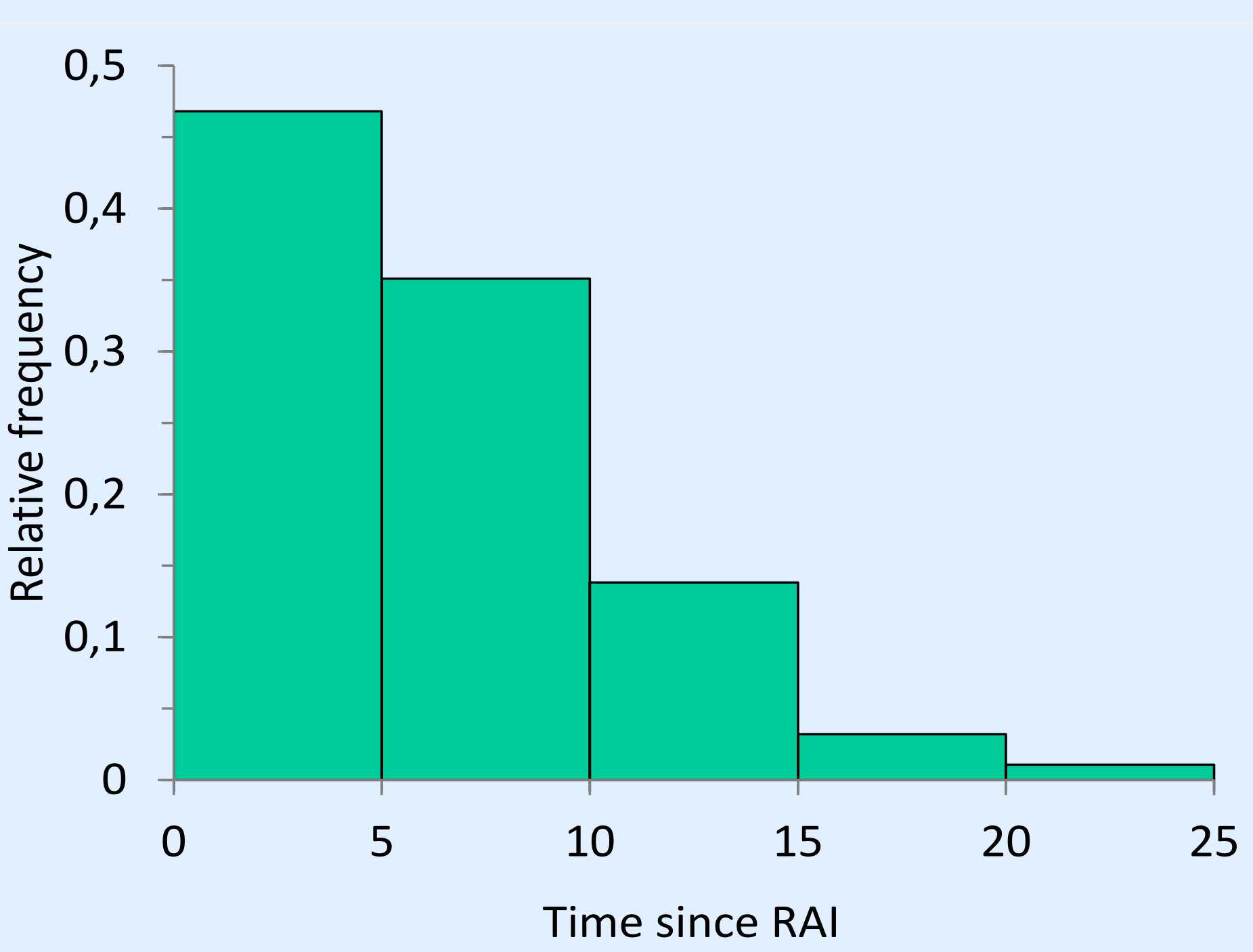

Figure 6. Time post RAl treatment in patients with a undetectable hs-Tg

\section{Current patient follow up}

- Patients with normal neck ultrasound and stimulated $\mathrm{Tg}<2 \mathrm{ng} / \mathrm{mL}$ are classified as low risk and have annual follow up for 5 years.

Proposed new patient follow up

-All patients to undergo dynamic risk stratification at 9-12 months post RAI.

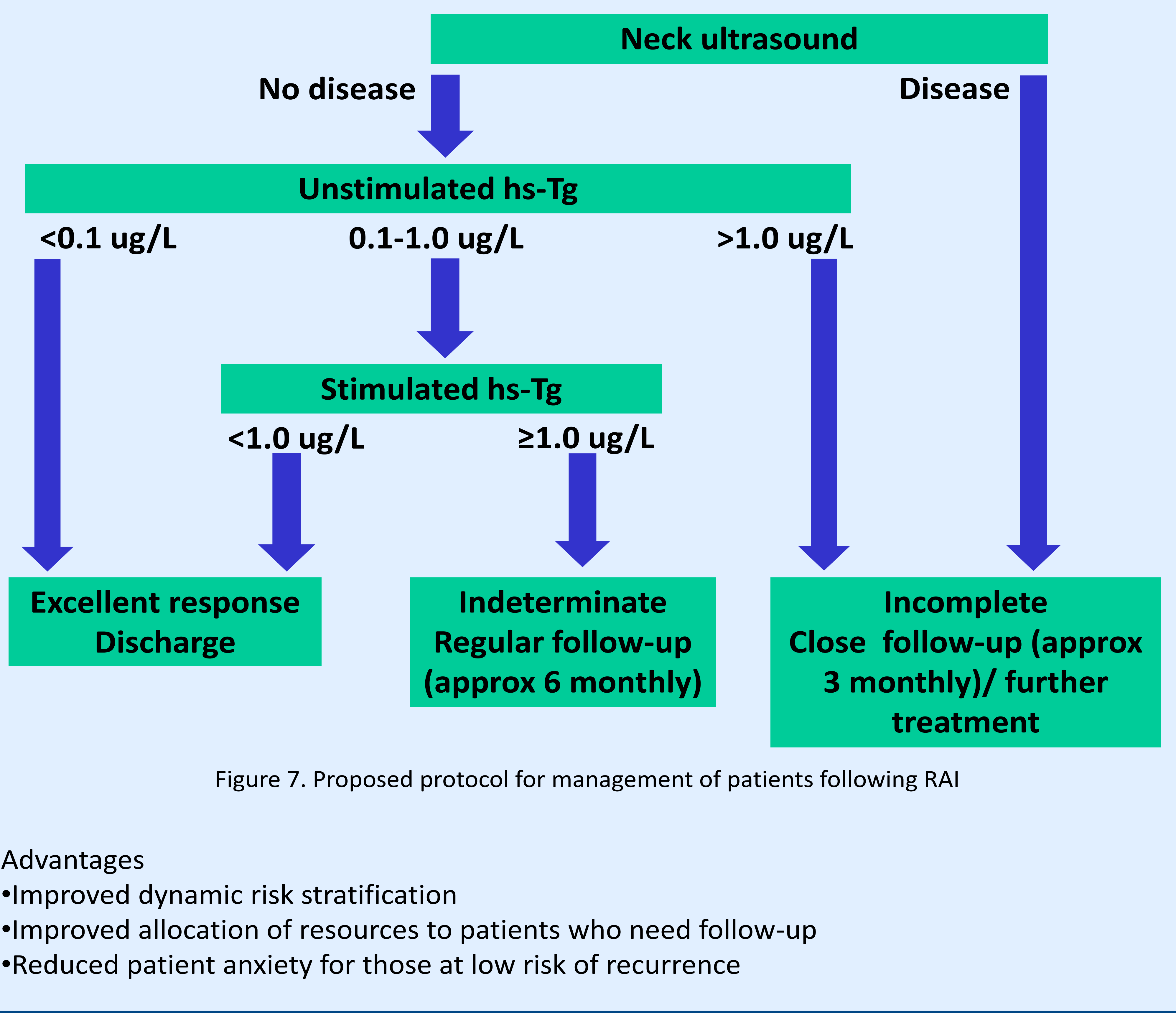

\section{Conclusion}

- For patients with ongoing follow-up, a period of paired analysis would be required due to the significant negative bias observed - The hs-Tg assay has acceptable imprecision and EQA performance.

-A subset of patients with detectable hs-Tg were identified; further investigation is required to determine the clinical significance.

-Discussions are ongoing with clinicians as to how hs-Tg would change practice.

\section{References}

1. 2015 American Thyroid Association Management Guidelines for Adult Patients with Thyroid Nodules and Differentiated Thyroid Cancer DOI: 10.1089/thy.2015.0020 